\title{
Point Mutations in the 90-kDa Heat Shock Protein Binding Region of the Glucocorticoid Receptor Affect the Functional Characteristics of the Receptor $^{a}$
}

\author{
CLAUDIO A. CAAMAÑO, ${ }^{b, d}$ M. INÉS MORANO, ${ }^{b}$ \\ FRIEDRICH C. DALMAN, ${ }^{c}$ MARY T. HOVERSTEN, ${ }^{b}$ \\ STANLEY J. WATSON, ${ }^{b}$ WILLIAM B. PRATT, ${ }^{c}$ AND \\ HUDA AKIL ${ }^{b}$ \\ ${ }^{b}$ Mental Health Research Institute \\ and \\ ${ }^{c}$ Department of Pharmacology \\ University of Michigan Medical School \\ Ann Arbor, Michigan 48109
}

Heat shock proteins (hsp) are a family of highly conserved and universally expressed proteins that are induced in all organisms and cultured cells by a variety of stressors. These stress proteins are considered to act as molecular chaperones that facilitate the correct protein folding in vivo. ${ }^{1}$

It is now known that steroid receptors are associated to hsps as heteromeric complexes. In the case of the glucocorticoid receptor (GR), the receptor complex is generated upon reaction of the steroid binding unit with a large cytosolic chaperone complex containing hsp90, hsp 70 and hsp56. Interestingly, the association of GR to at least hsp90 is required for the adoption of a high steroid-binding affinity state. ${ }^{2}$

We previously demonstrated that the hsp90-binding site is located within the hormone-binding domain of the mouse GR, supporting a two-site model in which the critical contact site occurs in the region of amino acids 632-659. This region contains a short proline-containing hydrophobic segment and adjacent dipole-pluscysteine motif that are conserved among all of the hsp90-binding receptors in the superfamily. A second hsp90 contact site was predicted in region 574-632, which contains the only highly conserved amino acid sequence in the receptor superfamily outside the DNA-binding domain. ${ }^{3}$

In order to further understand the structural requirements for the appropriate functioning of GR, we designed point mutations in the hsp90-binding region of the

\footnotetext{
${ }^{a_{\text {This }}}$ work was supported by Grants NIDA RO1-DA02265, NIMH PO1-MH42251-06, and NIADDKD P30-AM34933.

${ }^{d}$ Address correspondence to Dr. Claudio A. Caamaño, Mental Health Research Institute, University of Michigan. Ann Arbor, MI 48109-0720.
} 
rat GR (rGR). In one of the mutants, Pro-643 was changed to Ala (GRM0). To modify the above mentioned dipole Glu-649 was replaced by Gln in GRM1. Finally, a double mutant was obtained changing Lys-597 to lle and Pro-600 to Leu in the conserved $\mathrm{N}$-terminal end of the hsp90-binding region (GRM8). We expressed wildtype and mutant rGRs in two separate recombinant systems. In a bacterial system we obtained purified fusion constructs containing the DNA- and steroid-binding domains of rGRs which were treated with rabbit reticulocyte lysate for the assembly in vitro of functional complexes as described by Caamaño et al. ${ }^{4}$ In mammalian Cos1 cells, we expressed full-length rGRs. The steroid binding affinity and specificity, hydrodynamic behavior and association with hsp90 of these GRs were assayed in both expression systems. Their DNA-binding characteristics upon activation were evaluated using the bacterial system. Finally, a transcriptional activation assay was conducted cotransfecting Cos-1 cells with the different GRs and a glucocorticoidresponsive reporter plasmid.

The GRM1 showed no differences from the wild receptor in any of the assays performed. In contrast, the steroid binding activity of GRM8 was reduced by more than a hundredfold, and its transcriptional activation capacity was completely abolished. Finally, the GRM0 exhibited lower steroid-binding affinity than the wild-type control without modification in the steroid binding specificity. The hydrodynamic behavior was also different between GR and GRM0. While steroid-bound GR complexes migrated as $9 \mathrm{~S}$ forms under nonactivating conditions and as $4 \mathrm{~S}$ forms under activating conditions, more than $50 \%$ of the GRM0 complexes migrated as $4 \mathrm{~S}$ forms under nonactivating conditions. In addition, in the transcriptional activation assay GRM0 showed only $30 \%$ of the hormonal response of the wild type receptor.

In summary, we have demonstrated that within the hsp90-binding region of GR (amino acids 642 to 671 in the rat), Pro-643 (GRM0), but not the adjacent dipole (GRM1), is important for the in vivo GR function. In addition, in the other highly conserved hsp 90 contact site of GR (amino acids 586 to 642 in the rat) the change of Lys-597 and Pro-600 (GRM8) produces a dramatic decrease in steroid binding and abolishes the transactivation capacity of GR.

We conclude that the hsp- 90 binding region of GR is involved in the steroidbinding and the transactivation functions of the receptor complex.

\section{REFERENCES}

1. Hendrick, J. P. \& F.-U. HARTL. 1993. Molecular chaperone functions of heat shock proteins. Annu. Rev. Biochem. 62: 349-382.

2. PratT, W. B. 1990. Interaction of hsp90 with steroid receptors: organizing some diverse observations and presenting the newest concepts. Mol. Cell. Endocrinol. 74: C69-C76.

3. Dalman, F. C., L. C. Scherrer, L. P. Taylor, H. Akil \& W. B. Pratt. 1991, Localization of the $90-\mathrm{kDa}$ heat shock protein-binding site within the hormone-binding domain of the glucocorticoid receptor by peptide competition. J. Biol. Chem. 266: 3482-3490.

4. Caamaño, C. A., M. I. Morano, P. D. Patel, S. J. Watson \& H. Axil. 1993. A bacterially expressed mineralocorticoid receptor is associated in vitro with the 90 -kilodalton heat shock protein and shows typical hormone- and DNA-binding characteristics. Biochemistry 29: $1880-1886$. 